\title{
Haptically Enabled Handheld Information Display with Distributed Tactile Transducer
}

\author{
Jérôme Pasquero, Joseph Luk, Vincent Lévesque, Student Member, IEEE, Qi Wang, Member, IEEE, \\ Vincent Hayward, Senior Member, IEEE, and Karon E. MacLean, Member, IEEE
}

\begin{abstract}
This paper describes the design, construction, and initial evaluation of a handheld information device that supports combined tactile and graphical interaction. The design comprises a liquid crystal graphic display co-located with a miniature, low-power, distributed tactile transducer. This transducer can create electronically-controlled lateral skin deformation patterns which give the sensation of sliding over small shapes. It is integrated within a slider mechanism to control scrolling. It also functions as a detent when pushing on it. Tactile feedback and the combination of visual and tactile feedback in a mobile context enable the development of new functions, such as multimodal navigation within large graphic spaces.
\end{abstract}

Index Terms-Handheld information devices, tactile displays, haptic interfaces, multimodal interaction, tactile transducers, mobile devices.

EDICS Category: 3-INTF User Interfaces, 2-DISP Display Technology for Multimedia, 7-CONS Consumer Electronics and Entertainment, Joint Media and Multimodal Processing

\section{INTRODUCTION}

Artificial tactile feedback provides an underutilized channel which can be used to gain information about the state of a device, the occurrence of an event, or its content. In mobile devices, these possibilities have been exploited so far in a very limited way for want of adequate transducers.

In general, diverse approaches have been proposed as operating principles for tactile transducers, the electromechanical subsystem of a complete tactile display. These approaches differ by the actuator technology employed, by the manner in which tactile signals are delivered to the skin, by the type of tactile signals they produce, by practical factors such as bulk and power, as well as by the range of sensations that they can mediate. Many principles able to produce movement in a small space have been explored, including miniature solenoids that vibrate steel membrane elements by variable reluctance [1], surface acoustic waves that modify the sliding interaction between a surface and a plate [2], electrogel lamellae that push against the skin [3], and pneumatically-activated dimples rising out of a surface [4]. A more complete survey of tactile display technologies can be found in [5].

J. Pasquero, V. Lévesque, Q. Wang, and V. Hayward are with the Haptics Laboratory, Center for Intelligent Machines, McGill University, 3480 University Street, Montréal, PQ, Canada, H3A 2A7 (email: \{jay,vleves,qiwang,hayward\}@ cim.mcgill.ca). J. Luk and K. MacLean are with the Sensory Perception Interaction Group, Department of Computer Science, The University of British Columbia, 201-2366 Main Mall, Vancouver BC, Canada, V6T 1Z4 (e-mail: \{luk,maclean\}@cs.ubc.ca).
The list of designs attempted to date is truly great. The most common approach to making tactile transducers is to pack a dense array of actuated pins; an early example of which can be found in [6]. At rest, the pins resemble a flat surface. The display is activated by moving the pins normally to the skin to approximate a shape, much the way a graphical display approximates an illumination field with an array of pixels. However, this type of transducer has significant limitations including the difficulty of achieving a high density array, high power consumption, and high manufacturing cost. Due to these challenges, the resulting devices are typically complex (e.g. [7]) or not portable.

Compact designs are required for tactile displays to enter the mobile device arena. This is of particular importance for the development of synthetic, abstract tactile signals, such as haptic icons [8], and tactons [9], which aim at alleviating, through touch, the attentional and cognitive load imposed on users by human-computer technology. For instance, portable devices typically have a multitude of functionalities and are often used in demanding multitasking situations. Distributed skin stimulation delivered by integrated tactile displays can help create expressive and pleasant percepts capable of delivering substitutive or complementary information to users.

\section{A. Tactile Displays in Portable Devices}

The Optacon, a sensory-substitution device for the blind developed in the $70 \mathrm{~s}$, is a key example of an early portable system. It is made of a dense array of 144 skin contactors configured in a gutter geometry to maximize the amount of skin being stimulated [10]. Each contactor is on-off in that it can either vibrate at full amplitude or remain still. When connected to a camera held in one hand, it is possible - with training — to distinguish printed high-contrast patterns such as letters or line drawings while scanning them with the camera. This results in binary high-contrast tactile images, or tactile representations of printed material. Since then, other variations on this principle have been investigated such as the VITAL [1], which achieves an exceptionally slim form factor.

Another promising approach to portability is electrostimulation by which the skin is electrically coupled to an array of electrodes in order to create small currents in the superficial skin layers. This principle was the basis of early portable tactile displays for aiding the visually impaired [11]. There are many possible variants in current waveforms, electrode geometries, and skin coupling methods. One of the latest designs is described in [12]. 
Today, three types of portable tactile stimulators are commercially available. The first is the Braille reader for the visually impaired, e.g. [13]. The second is the vibrator found in portable phones, pagers, and game controllers by which a motor spins an eccentric mass to shake the supporting structure. The third is the voice-coil transducer which applies vibrotactile stimulation to the skin as an aid for the deaf to lip read. ${ }^{1}$

While having been adopted with some success by the commercial market, these tactile devices suffer from many limitations. Braille readers, which have pins that rise above a surface to produce Braille dots, are usable only for rendering refreshable Braille. Vibratory signals delivered by portable devices and voice-coils are spatially diffuse. They underexploit the human ability to perceive, discern, and process intricate distributed skin deformation patterns. Although recent industrial developments have enabled a wider variety of waveform patterns, ${ }^{2}$ the predominant sensation is still that of "buzzing".

\section{B. Distributed Lateral Skin Deformation}

Vibrotactile feedback will probably always have a role to play in information displays as a mechanically simple way to demand a user's attention. However, the sensations delivered by devices that vibrate evoke a distant and unnatural computer world. A likely explanation is that most ecologically familiar vibrotactile sensations arise from human-made objects, such as rotating machinery.

While it is unlikely that tactile displays will soon be able to convey the gamut of real-life tactile sensations, we believe that it is important to investigate tactile interaction techniques which feel natural and therefore, are better suited to the human reality. Touch feedback resulting from distributed skin deformations is potentially richer in content and form than vibrotactile stimulation.

Over the past few years we have developed a family of devices that operate according to a principle that departs from the conventional skin indentation approach [14]-[16]. Instead, tactile sensations are created by deforming the skin laterally, with negligible normal deflection. Because this display technique works by causing lateral skin deformations, we assign it the neologism laterotactile. A variety of sensations can be created this way, for example the sensation of sliding a finger over small shapes. We have recently succeeded in using this technique to display virtual Braille dots [17], as well as tactile graphics [18]. Because of its potential for miniaturization, laterotactile technology is a natural candidate for the integration of tactile displays in mobile devices.

\section{Paper Overview}

We explore the design, prototyping, and preliminary evaluation of a handheld device called the 'Tactile Handheld Miniature Bimodal device' (THMB, pronounced "thumb"). It features a tactile display mounted behind a liquid crystal

\footnotetext{
${ }^{1}$ See Audiological Engineering Corp. Tactaid ${ }^{\mathrm{TM}}$ - www.tactaid.com.

${ }^{2}$ See Immersion Corp. VibeTonz ${ }^{\mathrm{TM}}$ product literature.
}

display (LCD). This device enables the manipulation of information available visually on the screen as well as tactually on a transducer in contact with the user's left thumb (Fig. 1). The 'haptic interface' comprises a sliding active surface which is able to create a range of programmable tactile sensations. A miniaturized laterotactile display makes this possible.

In the remainder of this paper, we introduce the THMB and its interface, then describe its construction with sufficient detail to allow re-implementation. We close with an illustrative application example and a discussion of practical considerations. Several other scenario examples are discussed in [19].
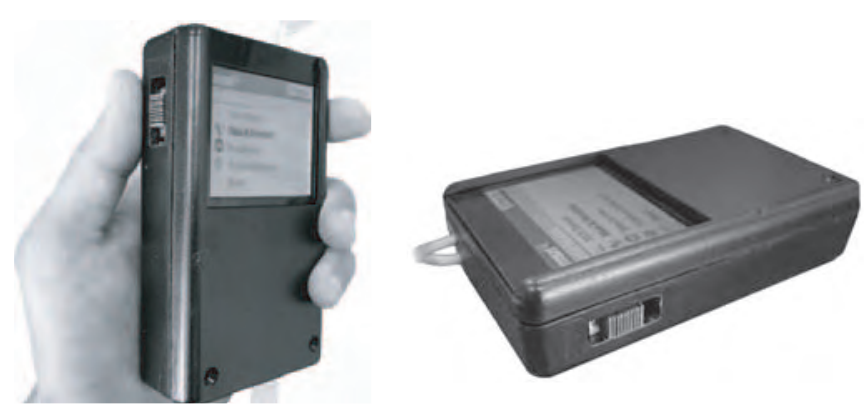

Fig. 1. The THMB device.

\section{The THMB: Designed For Bimodality AND ERGONOMICS}

The overarching design goal was to support tactile feedback correlated with control movements, while being sensitive to ergonomic considerations. We achieved the synergistic association of sensory-motor functions in a small space and with few resources by integrating a laterotactile display within a thumb-operated slider on the left side of a hand-sized case, and locating it directly under the LCD screen. Complementary modes of interaction can be supported this way, taking advantage of the human ability to process and integrate information in parallel [20].

Because of its hyper-agility, the thumb enjoys a unique status among fingers [21]. Its joints and musculature allow it to dexterously reach much of the inside of the hand. Many mobile devices rely on it for data input, for dialing, for writing text messages, or for browsing the web. The thumb also often operates scroll wheels positioned on the left side of a case, thereby freeing the right hand for other tasks. With the THMB device, the thumb operates the slider while the palm and remaining fingers hold the device at a convenient distance from the eyes (Fig. 2(a)). Several other hand postures may also be employed.

The tactile display is programmed to cause tactile sensations in the thumbpad as exemplified in Fig. 2(b). These sensations can be related to what is seen on the screen or they can convey signals on their own. The active surface of the tactile display protrudes slightly from the left side of the case, making it easy to slide the unit up and down by flexion of the thumb's distal phalanx. This mechanism also operates as a push-button triggered by light inward pressure. 


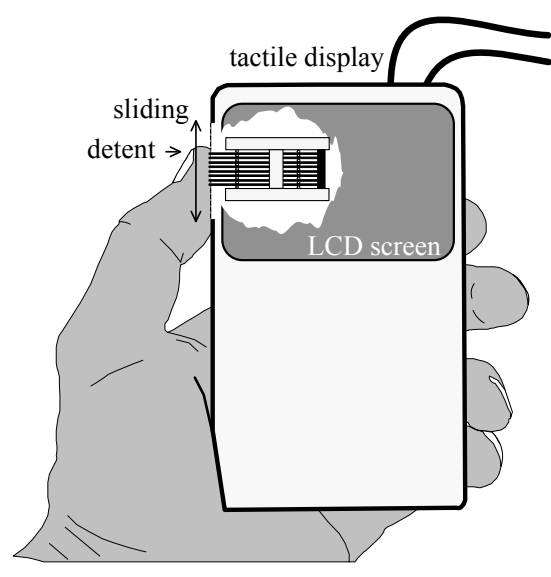

(a)

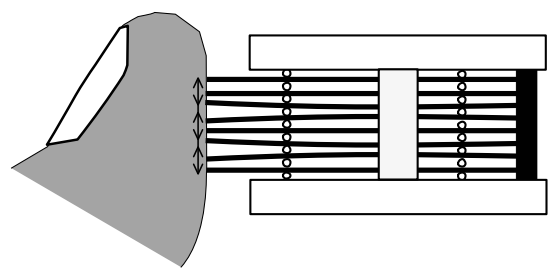

(b)

Fig. 2. (a) Overview of the THмB interface and (b) close-up of the tactile display stimulating the skin by lateral deformation.

\section{SPECIFYING DISTRIBUTED LATERAL SKIN DEFORMATION WITH THE THMB}

With distributed lateral skin deformation, a progressive wave of local tangential deformation in the fingertip skin gives the sensation of a small shape sliding on the finger [14]. Clearly, there are many ways to specify moving skin strain patterns, which vary in perceptual realism and distinctness. One technique that we have used in the past has patterns of deflection such that for one actuator $i$, the deflection $d_{i}$ is:

$$
d_{i}\left(y_{s}, t\right)=P\left(y_{s}+(i-1) \epsilon, t\right), i \in[1 \cdots N]
$$

where, referring to Fig. 3, $N$ is the total number of actuators, $t$ is time, $\epsilon$ is a spatial period preferably equal to that of the display, about $0.9 \mathrm{~mm}$ for the THMB, and $P($.$) is an admissible$ function. The variable $y_{s}$ stands for the position of a slider on which the display is mounted; it can also serve as a simulated position if no slider is available.

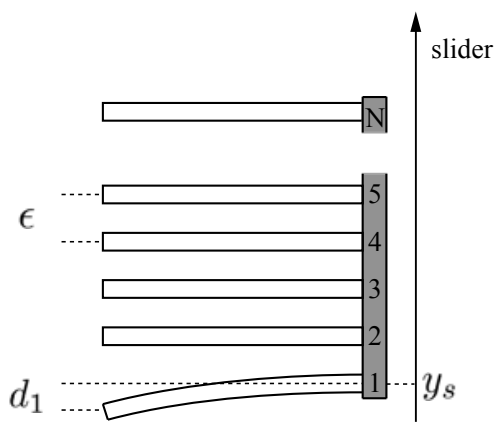

Fig. 3. Variables for the deflection function of Eq. (1).
Simple deflection patterns do not depend on time and must be explored actively. One example is a sinusoidal grating $d_{i}\left(y_{s}\right)=A \sin \left(\left[\pi\left(y_{s}+(i-1) \epsilon\right)\right] / 2\right)$. Other important cases consist of features traveling automatically at constant speed. Fig. 4 shows five actuators undergoing a sequence of deflections and their consequences on the deformation of the skin for a feature moving upward.
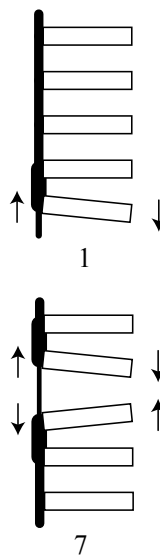

7
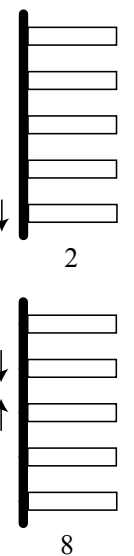

8

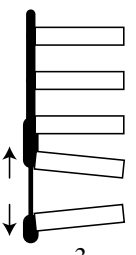

3

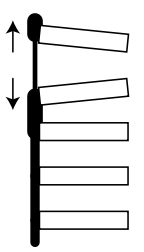

9

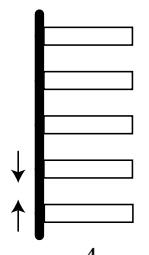

4

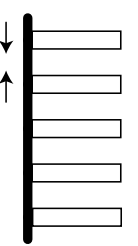

10
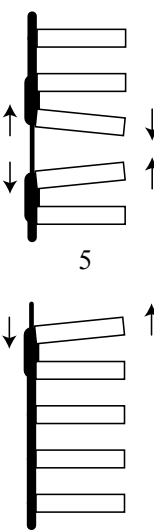

11

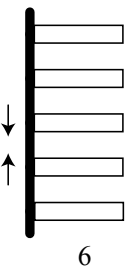

6

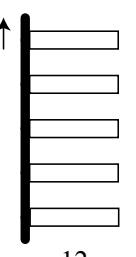

12
Fig. 4. Example of a moving feature with the slider fixed. Only key frames are shown. In practice transitions are smooth.

Generally, when the finger first comes in contact with a static undeflected display surface (e.g. frame 12 in Fig. 4), the skin is in a state of nearly neutral strain. The initial sensation is that of a flat surface because the actuators are sufficiently dense and the receptors embedded in the skin respond to deformation [22]. The sensation of a moving feature corresponds to traveling waves with specific deformation trajectories. In such instances, an individual patch of skin could be first in a relaxed state. It would then expand, relax, compress, and become relaxed again. In the meantime, the neighboring patch would undergo the same trajectory, but with a phase difference. To employ a physics terminology, if the phase velocity is equal to the group velocity, then a given fixed pattern will be felt to be moving at that velocity. If this velocity is equal and opposite to that of the slider, then the pattern is felt to be stationary with respect to the THMB enclosure. The THMB supports more complex functions which are left for future studies.

\section{System DesCRIPTION}

The transducer can easily be manufactured as a powerefficient, compact unit having a low component count following a technique inspired from earlier devices [15], [17]. Piezoelectric actuators can be inexpensive, light, powerefficient, and in fact are already in common use in many portable devices, including refreshable Braille displays and mobile phones. The technology is mature, well-understood, and steadily improving. Therefore, it is well suited for portable devices and allows for tactile displays with high spatial resolutions that require only one single part per actuator. On the downside, piezoelectric actuators are limited in the displacement they yield and require high activation voltages.

In this section, we seek to provide sufficient details for the replication of the THMB display, given access to modest fab- 


\section{IEEE Transactions on Multimedia, 2007, 9(4):746-753}

rication facilities and, whenever possible, using off-the-shelf components with a view to demonstrate practical viability.

\section{A. Piezoelectric Benders}

The display makes use of piezoelectric bimorphs, also called benders, to generate lateral skin deformation. A bimorph bender has two layers of piezoelectric material bonded to a central electrode, plus electrodes coated on the outer faces. When an electric field is created in these layers with proper polarization, they experience opposing dimensional changes. The stress distribution created by the common boundary results in bending deformation of the entire slab [23]. The dynamic constitutive relationships are complex, but at frequencies below the first resonant mode, the electrical behavior of an actuator is well approximated by that of a capacitor. This means that when operating under a dc voltage, an actuator remains bent but consumes no power.

Benders are usually mounted in a cantilever configuration as shown in Fig. 5(a), but in the тнмв, they are stacked between two rows of thin rods as illustrated in Fig. 5(b). This configuration yields a displacement-stiffness tradeoff that differs significantly from that of ordinary cantilevers. For a given free deflection, this structure will always be stiffer, but have a smaller deflection [24]. A high stiffness is essential because skin is a tough medium to deform. The dual-pinning mount allows actuators to be smaller and to operate from lower voltages for an equivalent resulting tactile stimulation.

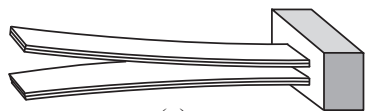

(a)

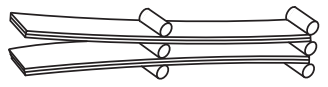

(b)

Fig. 5. Two possible configurations for the piezo-actuators: (a) cantilever mount and (b) stiffer dual-pinning mount used for the ТнMB.

\section{B. Preparation of the Benders}

Raw piezoelectric sheets obtained from the manufacturer are first prepared to provide for electrical connections compatible with the tight spacing requirements. The preparation is analogous to the fabrication of microelectronic circuits: a sequence of deposition and removal of material. Fabrication can be done in an ordinary mechanical workshop rather than in a cleanroom, and tools are a temperature-regulated soldering station, a precision knife, a pair of scissors, and model painting brushes. The materials are piezoelectric bender plates (eight units from Piezo Systems $₫$ T215-H4-203Y), conductive tape (3M Corporation, EMI Copper Foil Shielding Tape 1181), standard nonconductive adhesive tape, conductive paint (Loctite $\AA$ Quick Grid ${ }^{\mathrm{TM}}$ found in car rear window defogger repair kits, product \#15067), and polyurethane varnish.

As illustrated by Fig. 6(a), the slabs are first coated with tracks of conductive paint to improve their resistance to scratches. The two external electrodes are then electrically connected using strips of conductive tape wrapped around the plates. The conductive strip adheres directly to the external electrodes so patches of non-conductive tape must first be applied to prevent contact with the central electrode. To provide access to the central electrode, soldering pads are created by grinding off channels in the external electrodes. These pads are connected to the central electrode using a small amount of conductive paint applied on the side faces to which a thin wire can then be soldered. The resulting solder joint must be thinner than the space between two neighboring actuators. Finally, the tips of the plates are coated with several varnish layers to isolate them from the user's skin.

\section{Transducer Assembly}

The tactile display prototype is made of eight 0.38 -mm-thick piezoelectric benders (6.4-by-31.8 $\mathrm{mm}$ ), sandwiched between 2 rows of 0.5 -mm-diameter brass rods that act as hinges of a dual-pinning configuration (see section IV-A) and as ground connections. Two passive brass plates at the ends protect the benders from excessive lateral stress applied by the user's thumb (Fig. 6(b)). The assembly is held together between two aluminum clamps secured with four screws that act as support for the rods. Once assembled, the transducer constitutes a single unit where only the actuator tips are free to move. In the present design, the rods are located at $5 \mathrm{~mm}$ and $23 \mathrm{~mm}$ respectively from the bottom of the actuator. The benders rest at the bottom on a plastic locating pin. Once the transducer is assembled, as shown on Fig. 6(c), the size of the exposed contact area is about $8.7 \mathrm{~mm}$ by $6.4 \mathrm{~mm}$. During the assembly phase, locating pins (not shown) secure the piezoelectric benders alignment along the display axis.

\section{Integration Inside the Case}

The basic transducer is integrated in a ready-made case in order to provide for a sliding mechanism combined with a push-button. As seen in Fig. 7(a), two parallel guiding stainless steel shafts connect two parallel plates, and pass through the assembled transducer's two sleeves. Structural rigidity is provided by a strut that also supports a linear potentiometer. The axis-to-axis distances of the two steel shafts, the transducer's sleeves, and the potentiometer strut are all matched with exacting precision in order to guarantee smooth operation. Sliding travel is limited by spacers to a distance of $11 \mathrm{~mm}$. This results in the sub-assembly shown in perspective view in Fig. 7(b).

The sub-assembly is inserted in an off-the-shelf plastic case (Hammond $\AA 1593$ series instrument enclosure). Such prototyping boxes typically provide slots to secure printed circuit boards. As seen in Fig. 8, we use these to anchor a pivoting mechanism that transfers the detent behavior of a miniature, board-mountable switch to the tactile display surface itself. This way, the THMB device is given a slide-andpress-detent behavior similar to that of a scroll wheel with the difference that the tactile sensations associated with scrolling are not fixed but programmable in a number of ways. 


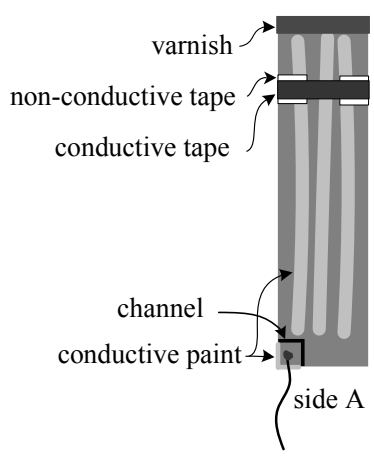

(a)

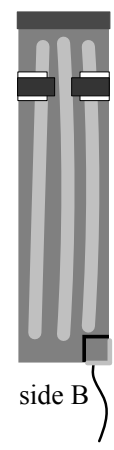

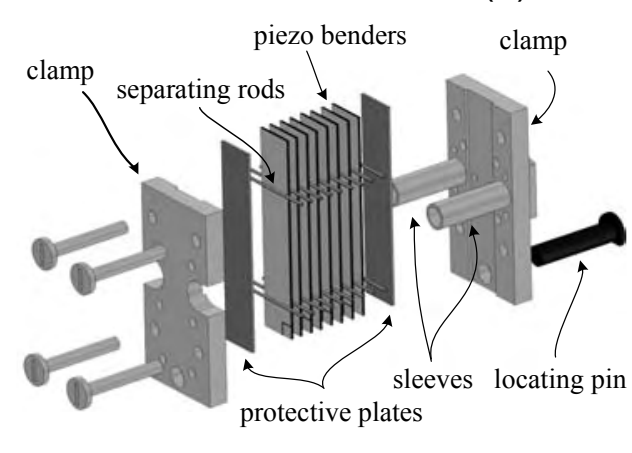

(b)

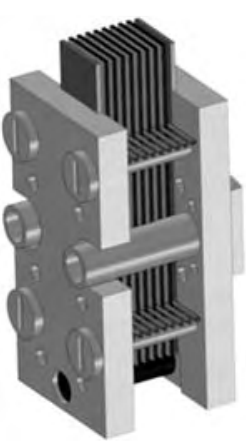

(c)

Fig. 6. Building the transducer: (a) preparation of the piezoelectric actuators, (b) assembly and (c) ready to be mounted to the slider.

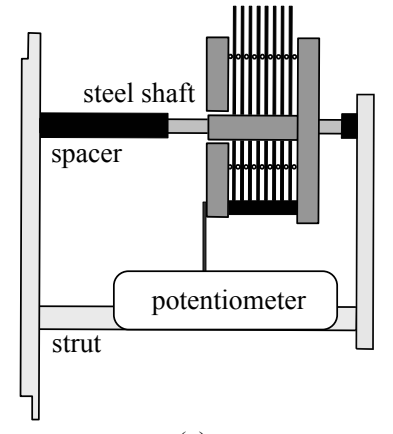

(a)

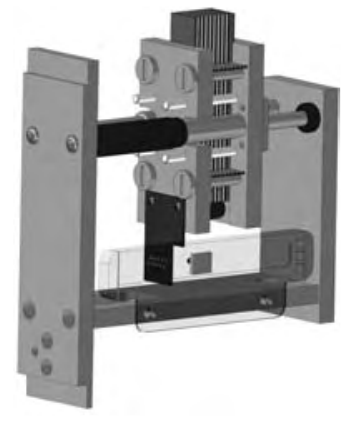

(b)

Fig. 7. Sliding mechanism: (a) $2 \mathrm{D}$ schematic of the sliding mechanism and (b) $3 \mathrm{D}$ view of the integrated system.

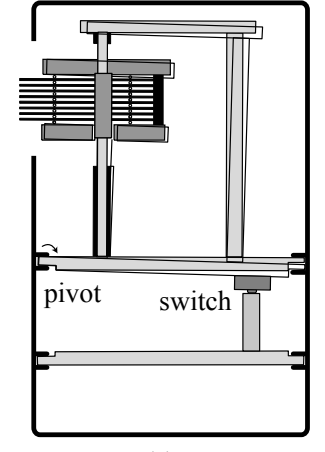

(a)

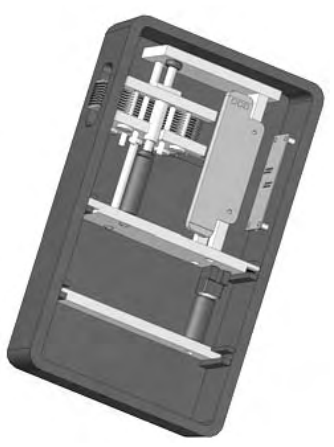

(b)

Fig. 8. (a) Integration of the sub-assembly in prototyping box and (b) 3D view of the integrated system.

The large number of connections packed in a small space makes for a significant wire management problem. A strategy similar to that used in electromechanical consumer devices such as printers and scanners is employed. Referring to Fig. 9, two miniature printed circuit boards, one moving $(\mathrm{PC}-1)$ and one fixed ( $\mathrm{PC}-2)$, act as concentrators. A $0.5 \mathrm{~mm}$ flat flex cable (from Parlex $\AA$ ) allows for a smooth and reliable connection. Two round, miniature-gauge silicone cables (Cooner Wires $®$ ) AS323REV1) route the 14 signals to external electronics.

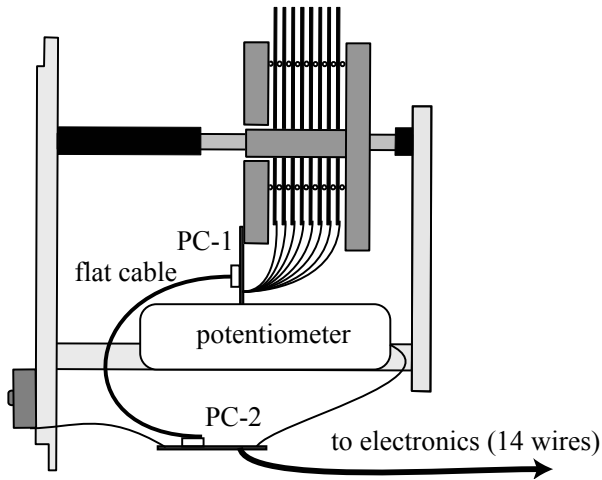

Fig. 9. Wire management.

\section{E. Electronics}

For the purpose of this prototype, the power and control electronics were adapted from earlier projects [15], [17], and were kept external to the casing. Briefly, the digital system comprised a personal computer (PC) and a field-programmable gate array (FPGA) development board (Constellation board ${ }^{\mathrm{TM}}$ $10 \mathrm{~K} 50 \mathrm{E}$ from Nova Engineering $($ ) ) supporting universal serial bus 1.1 (USB) connectivity (Fig. 10).

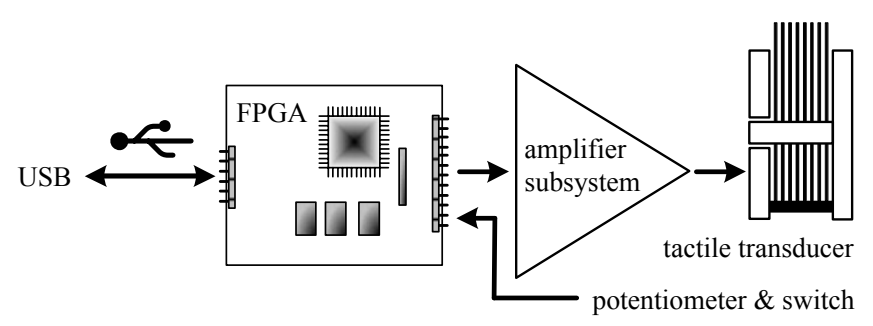

Fig. 10. Overview of THMB electronics.

The FPGA is programmed to convert the serial data coming from the personal computer to eight parallel $156.25-\mathrm{kHz}$ pulsewidth modulation (PWM) output signals. The eight digital level PWM signals are fed to eight analog comparators that step up the voltage to $\pm 15 \mathrm{~V}$. The resulting signals are low-passed and supplied to eight high-voltage operational amplifiers (BurrBrown $($ OPA 445) which in turn drive the eight benders at analog levels between $\pm 45 \mathrm{~V}$ (Fig. 11). The FPGA also 
reads the output of an analog-to-digital converter detecting the position of the slider, and provides this information, along with the state of the switch, to the personal computer.

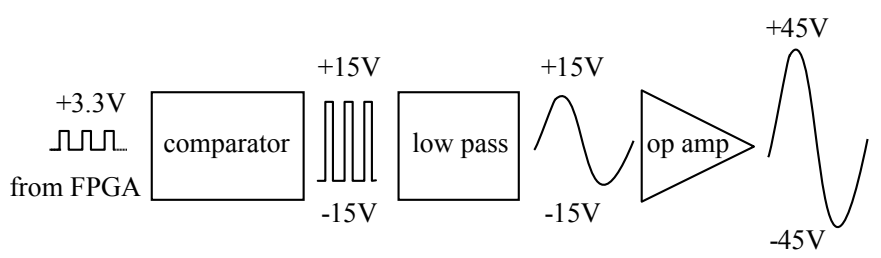

X 8

Fig. 11. Operation over one of the eight channels of the amplifier.

\section{F. Liquid Crystal Display}

The Тнмв is equipped with a $58.8 \times 49.9 \times 5.8 \mathrm{~mm}$ color LCD screen inserted in the casing, along with its control electronics. The LCD system accepts NTSC format signals and therefore, computers with a TV-out port can drive the LCD screen as an external monitor. A second miniature-gage flexible cable connects the THMB's screen to the computer's video-out port.

\section{G. Software}

The THMB's host software was written using the STRESS Software Development Kit (STRESSd, pronounced "stressed"). STRESSd consists of a cross-platform C++ object library for Linux and Microsoft Windows ${ }^{\mathrm{TM}}$. It provides a simple, common interface to most of our laterotactile displays (STRESS [15], VBD [17], and THMB). The library provides a layered interface allowing interaction with the displays at different levels of abstraction. Low-level interaction with the FPGA development board occurred through a separate Software Development Kit that we have released under an open-source license. $^{3}$

\section{Preliminary Evaluation: 'Scroll-And-Feel'}

In the context of mobile interaction, users' cognitive resources are dedicated, in order of importance, to acting on the environment (e.g., walking, talking), to monitoring it (e.g., to scan the environment visually), and lastly to interacting with the mobile device [25]. For this reason, an undivided attention to the screen cannot be expected. During interactions such as browsing the web on a mobile phone, tactile feedback can provide continuous bridging information between glances at the screen.

With this in mind, a preliminary application was developed to exercise the device's capabilities, with the goal of implementing concurrent tactile and visual exploration of a graphical document that is too long to fit on the screen, and has to be scrolled up and down by using the slider.

\footnotetext{
${ }^{3}$ Sidus - http://sidus.sourceforge.net.
}

\section{A. Programming}

a) User Control: Under velocity control, it is possible to browse through an entire image by controlling speed and direction. The displacement range of the slider is divided into three zones. When the slider is inside a central neutral zone, the graphical image does not move. Moving the slider away from the center causes the image to scroll up or down at a speed proportional to the deflection from the center. Absolute positioning is applicable only within a small scope.

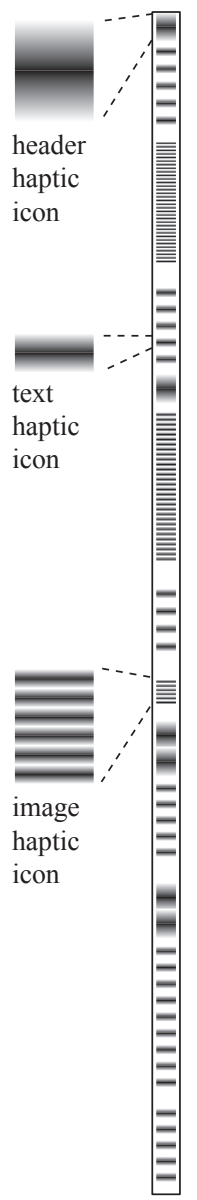

(a)

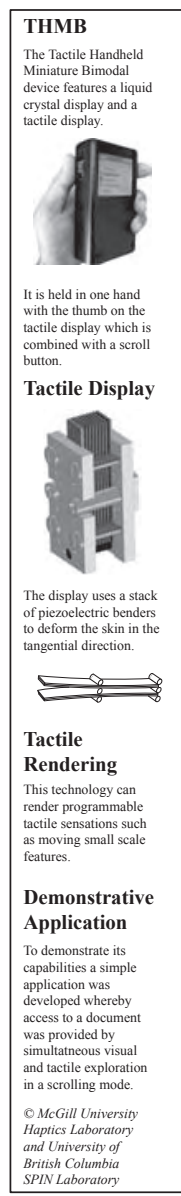

(b)

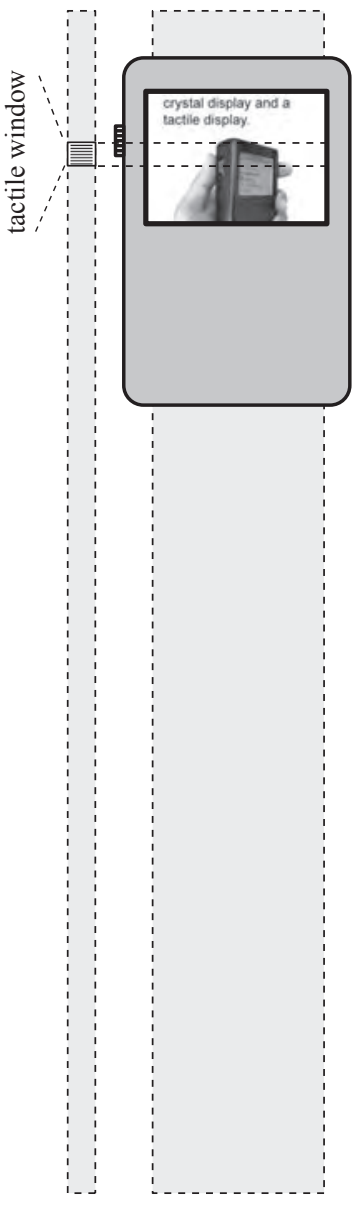

(c)
Fig. 12. Scrolling application for evaluation: (a) tactile track with the three types of haptic icons, (b) graphic track and (c) tactile window mapped to the center of the device's graphical window.

b) Tactile Mapping: There are many ways to map a scrolling graphical image to a tactile sensation. We adopted a simple strategy that associated a tactile track to a graphical track. The two are scrolled simultaneously under slider command. The graphical track contains short paragraphs of text separated by headers and small figures (Fig. 12(b)). The tactile track is composed of a series of tactile haptic icons, see Fig. 12(a). A specific dynamic tactile sensation is felt on the thumb skin when a given tactile icon travels across the tactile window, a subset of the graphic track currently visible on the LCD (Fig. 12(c)).

Haptic icons were created for the three types of tags found in the document: header, text and image. The three types of 


\section{IEEE Transactions on Multimedia, 2007, 9(4):746-753}

tactile sensations are spatial functions of the position of the corresponding haptic icon in the tactile window. Referring to the three icons in Fig. 12(a), light regions represent a downward deflection of the corresponding actuator, while dark regions represent an upward deflection. When the page is scrolled, a given haptic icon activates different benders, thus creating what can be described as peristaltic skin deformation such as the patterns that can be observed when a finger slides over a featured surface [26].
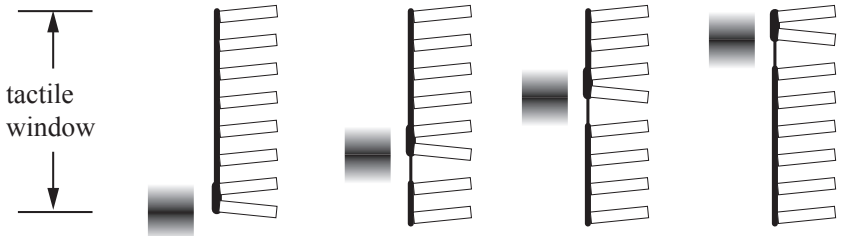

Fig. 13. Peristaltic deformation of the skin shown while a single line of text passes through successive frames of the tactile window (from bottom to top): position of the the text haptic icon within the tactile window (left column in each frame) and resulting skin deformation (right column).

This mapping and its effect are illustrated in Fig. 13 for a text haptic icon. In this case, a single element travels upward in the tactile window. The positions of the eight actuators are shown in successive frames where patches of skin between two actuators, i.e. taxels, are stretched and compressed. Because the strain experienced by the skin is the result of the differential deflection of adjacent actuators, the strain is related to the gradient of the grey level of a haptic icon representation. Adjacent frames indicate the skin's deformation trajectory when the image scrolls by a distance equal to the display's spatial period.

A text icon spans about half of the height of a line. It causes a full but single back-and-forth bending motion of an actuator. The resulting sensation of a text icon moving from actuator to actuator is often felt as a tiny, sharp, and isolated traveling feature. Header icons are widened versions of the text icons and therefore, they are more diffuse and of lower contrast. They are also often perceived to be weaker and larger. Finally, image icons consist of a texture spanning an image's height. This generates a sensation rich in high frequencies. During exploration, the overall impression is that of tactile flow when multiple icons travel across the thumb.

\section{B. Initial Reports}

Six volunteers, all mobile phone users in their 20 s with no previous experience with the Тнмв device were recruited. They were not compensated for their time. These participants freely explored the scroll-and-feel application and answered a few questions in 10-to-15-minute sessions. They were given no instructions other than the manner in which the device should be held.

The application represented by Fig. 12 was designed to be stylistically similar to news webpages found on the Internet. A red marker displayed at a fixed position on the left side of the graphical display delineated the smaller tactile window within the graphical window. This way, the participants knew what part of the document they were exploring tactually. The graphical page was formatted to fit the width of a portable screen. It was displayed on the device's LCD for the two first participants and emulated on a PC monitor for the other four because of a hardware failure. The window sizes were the same in both configurations.

Half of the participants reported that the tactile sensations during scrolling were like touching a traveling train of ridges under the thumb. The illusion of motion was robust. Most of the participants $(5 / 6)$, however, could not initially distinguish more than two different types of tactile sensations. The closely spaced ridges comprising the image icon and the loosely spaced text or header icons were so different that more subtle differences may have been obscured [8]. Upon further questioning, participants were eventually able to differentiate between them. Using this feedback, we were able to redesign the haptic icons to make all three clearly identifiable and differentiable. This development is beyond the scope of this paper and will be reported in the future.

A few participants mentioned that "playing" with the device was pleasant, but could not verbalize exactly why. Not surprisingly, some (3/6) compared the image icon to vibration and/or texture, probably because of its high spatial frequency content. On the other hand, all seemed to agree that the text and header icons were compelling and completely different from anything related to vibrotactile stimulation. In general, all participants welcomed the velocity control; one of them even commented on its value. The subjective responses showed no differences between setups. We collected enthusiastic comments; some participants even envisioned environments and situations where our device could be useful. These and others are further developed in [19].

Overall, the participants' ability to discriminate icons shows promise. It provides evidence that percepts richer than those currently available on handheld devices are possible in a mobile context.

\section{IMPLICATIONS FOR FEASIBILITY AND FUTURE WORK}

We have built what is to our knowledge the first realistic prototype of a mobile handheld interface capable of directcontact distributed tactile feedback. We hope that this tactile delivery technology will enable portable tactile displays in the same way that LCD technology has enabled portable visual displays. The key is lateral skin deformation, which utilizes piezoelectric technology in an efficient configuration for a compact, high-resolution tactile transducer.

The process of building the first generation THMB suggests that it will be feasible to incorporate the technology into consumer devices. In its present prototype form, the THMB is tethered, with remotized circuitry and power requirements. The dimensions and weight of the transducer still need to be reduced by a factor of two or three to meet the requirements for a practical mobile device. Nevertheless, nothing in the design makes these limitations terminal.

The participant's first impressions suggest that the ТнМв can convey compelling tactile sensations in a multimodal use environment, and these encouraging results motivate further investments. In [27], Chan et al. successfully identified perceptual groupings of haptic icons based on distinctiveness. 
Such a systematic technique should be adapted to future developments of the tactile sensations displayed on the THMB. We have also already embarked on application-specific studies of information manipulation in the small space available in a mobile device, and we will be further evaluating how directcontact tactile feedback can provide added value in terms of both performance and comfort.

\section{ACKNOWLEDGMENT}

This work was performed in part with support of the Fonds Québécois de la recherche sur la nature et les technologies (FQRNT), the Natural Sciences and Engineering Council of Canada (NSERC) and PRECARN/IRIS. The authors would like to thank Don Pavlasek for insights and expertise, Joe Boka and Bob Thomson for their technical support, Andrew Gosline for helpful comments and all participants in the initial reports for providing valuable feedback.

\section{REFERENCES}

[1] M. Benali-Khoudja, M. Hafez, J.-M. Alexandre, A. Kheddar, and V. Moreau, "Vital: a new low-cost vibro-tactile display system," in Proc. IEEE International Conference on Robotics and Automation, 2004. ICRA '04, vol. 1, New Orleans, LA, April 2004, pp. 721-726.

[2] M. Takasaki, H. Kotani, T. Nara, and T. Mizuno, "Transparent surface acoustic wave tactile display," in Proc. IEEE/RSJ International Conference on Intelligent Robots and Systems, 2005, pp. 1115-1120.

[3] M. Konyo, S. Tadokoro, T. Takamori, and K. Oguro, "Artificial tactile feel display using soft gel actutators," in Proc. IEEE International Conference on Robotics \& Automation, 2000, pp. 3416-3421.

[4] G. Moy, C. Wagner, and R. S. Fearing, "A compliant tactile display for teletaction," in Proc. IEEE Int. Conf. on Robotics and Automation, 2000, pp. 3409-3415.

[5] J. Pasquero, "Survey on communication through touch," Center for Intelligent Machines - McGill University, Tech. Rep. TR-CIM 06.04, 2006. [Online]. Available: www.cim.mcgill.ca/ $\sim$ haptic/publications.html

[6] C. L. V. Doren, D. G. Pelli, and R. T. Verillo, "A device for measuring tactile spatiotemporal sensitivity," J. Acoust. Soc. Am., vol. 81, no. 6, pp. 1906-1916, 1987.

[7] I. Sarakoglou, N. Tsagarakis, and D. G. Caldwell, "A portable fingertip tactile feedback array, transmission system reliability and modelling," in Proc. of the First Joint Eurohaptics Conference and Symposium on Haptic Interfaces for Virtual Environment and Teleoperator Systems WHC'05, 2005, pp. 547-548.

[8] K. MacLean and M. Enriquez, "Perceptual design of haptic icons," in Proc. Eurohaptics, Dublin, Ireland, 2003, pp. 351-363.

[9] S. A. Brewster and L. M. Brown, "Tactons: Structured tactile messages for non-visual information display," in 5th Australasian User Interface Conference (AUIC2004), Dunedin, Australia, 2004, pp. 15-23.

[10] J. Bliss, M. Katcher, C. Rogers, and R. Shepard, "Optical-to-tactile image conversion for the blind," IEEE Transactions on Man-Machine Systems, vol. 11, pp. 58-65, 1970.

[11] S. Tachi, K. Tanie, K. Komoriya, and M. Abe, "Electrocutaneous communication in a guide dog robot (meldog)," IEEE Transactions on Biomedical Engineering, vol. 32, no. 7, pp. 461-469, 1985.

[12] H. Kajimoto, M. Inami, N. Kawakami, and S. Tachi, "Smarttouch: Augmentation of skin sensation with electrocutaneous display," in Proc. of the 11th Symposium on Haptic Interfaces for Virtual Environment and Teleoperator Systems, 2003, pp. 40-46.

[13] O. Tretiakoff and A. Tretiakoff, "Electromechanical transducer for relief display panel," US Patent No. 4,044,350, 1977.

[14] V. Hayward and M. Cruz-Hernandez, "Tactile display device using distributed lateral skin stretch," in Proc. of the Haptic Interfaces for Virtual Environment and Teleoperator Systems Symposium (IMECE2000), vol. DSC-69-2. ASME, 2000, pp. 1309-1314.

[15] J. Pasquero and V. Hayward, "STRESS: A practical tactile display system with one millimeter spatial resolution and $700 \mathrm{~Hz}$ refresh rate," in Proc. Eurohaptics 2003, 2003, pp. 94-110.

[16] Q. Wang and V. Hayward, "High-performance distributed tactile display device based on lateral skin deformation," in Proc. Haptics Symposium 2006, IEEE, 2006, Washington, Maryland, USA, 2006, pp. 67-72.
[17] V. Levesque, J. Pasquero, V. Hayward, and M. Legault, "Display of virtual Braille dots by lateral skin deformation: Feasibility study," ACM Transactions on Applied Perception, vol. 2, no. 2, pp. 132-149, 2005.

[18] Q. Wang, V. Levesque, J. Pasquero, and V. Hayward, "A haptic memory game using the STRESS ${ }^{2}$ tactile display," in Proc. of the 2006 ACM Conference on Human Factors in Computing Systems, CHI 2006, 2006, pp. 271-274.

[19] J. Luk, J. Pasquero, S. Little, K. MacLean, V. Levesque, and V. Hayward, "A role for haptics in mobile interaction: Initial design using a handheld tactile display prototype," in Proc. CHI'06, ACM, Ed., 2006, pp. 171180.

[20] S. Oviatt, "Ten myths of multimodal interaction," Communications of the ACM, vol. 42, no. 11, pp. 74-81, 1999.

[21] F. R. Wilson, The hand: How its use shapes the brain, language and human culture. Random House, 1998.

[22] R. S. Johansson and A. B. Vallbo, "Tactile sensory coding in the glabrous skin of the human hand," Trends Neuroscience, vol. 6, pp. 27-31, 1983.

[23] J. G. Smits, S. I. Dalke, and T. K. Cooney, "The constituent equations of piezoelectric bimorphs," Sensors and Actuators A, vol. 28, pp. 41-61, 1991.

[24] Q. Wang and V. Hayward, "In vivo biomechanics of the fingerpad skin under local tangential traction," J. of Biomechanics, 2006, in press, available online on the journal website.

[25] A. Oulasvirta, S. Tamminen, V. Roto, and J. Kuorelahti, "Interaction in 4-second bursts: the fragmented nature of attentional resources in mobile HCI," in of SIGCHI'05 Conference on Human Factors in Computing Systems, A. Press, Ed., New York, 2005, pp. 919-928.

[26] V. Levesque and V. Hayward, "Experimental evidence of lateral skin strain during tactile exploration," in Proc. Eurohaptics 2003, 2003, pp. 261-275.

[27] A. Chan, K. E. MacLean, and J. McGrenere, "Learning and identifying haptic icons under workload," in Proc. of First Joint Eurohaptics Conf. and Symp. on Haptic Interfaces for Virtual Environment and Teleoperator Systems (WHC 2005, IEEE-VR2005), Pisa, Italy, 2005, pp. 432-439. 


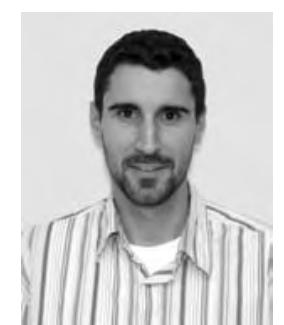

Jérôme Pasquero received the BEng and MEng degrees from McGill University, Montreal, Canada, in 2001 and 2003 respectively. Since 2001, he has been with the McGill University Haptics lab where he is currently pursuing his Ph.D. His research interests include tactile displays, Braille displays, tactile perception, human-computer interactions and tactile rendering for mobile devices.

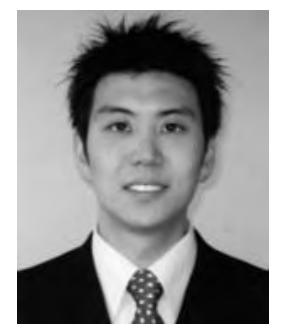

Joseph Luk received the BS degree in cognitive science from the University of California, San Diego in 1999, and the MSc degree in computer science from the University of British Columbia, Canada in 2006. $\mathrm{He}$ has worked as a mobile interaction designer for Pixo, Inc., in Silicon Valley, and continues to pursue interests in mobile user interface design, computer science education, and international / cross-cultural usability.

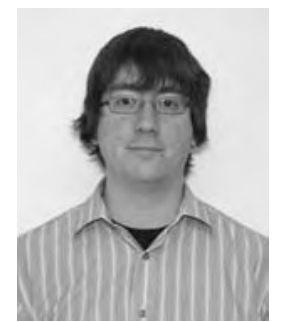

Vincent Lévesque received the BEng degree in computer engineering and the MEng degree in electrical engineering from McGill University (Montral, Canada) in 2000 and 2003 respectively. He has been with McGill's Haptics Laboratory since 2000 and is currently pursuing a Ph.D. His research interests include tactile displays and rendering, humancomputer interaction, and applications of haptics for persons with visual impairments.

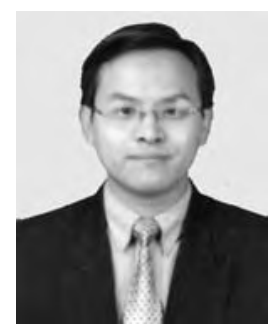

Qi Wang received his BEng degree in Mechanical Engineering from North China Electric Power University, China, in 1992, and the MEng and Dr.Eng degrees in Mechanical Engineering, and Mechatronics Engineering from Harbin Institute of Technology, China, in 1995 and 1998, respectively. Since September 2002, he has been pursuing his $\mathrm{Ph} . \mathrm{D}$. degree in Electrical and Computer Engineering at McGill University, Canada. His main research interests include human-machine interfaces, neural prosthetics, neural signal coding, and biomedical

instrumentation

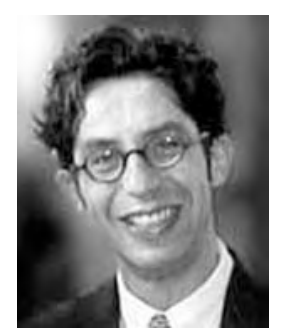

Vincent Hayward , Ing. École Centrale de Nantes; Ph.D. Computer Science 1981, Univ. of Paris. Chargé de recherches at CNRS, France (1983-86). Now Professor of Electrical and Computer Engineering at McGill University, he is interested in haptic interfaces design and applications, perception, and robotics. He is leading the Haptics Lab. at McGill and was the Director of the McGill Center for Intelligent Machines. Spin-off companies: Haptech (1996), now Immersion Canada Inc. (2000), RealContact (2002). Co-Founder Experimental Robotics Symposia, past Associate Editor IEEE Transactions on Robotics and Automation, Program Vice-Chair 1998 IEEE Conference on Robotics and Automation, Program Co-Chair ISR2000, Governing board Haptics-e. Past leader of a national project of IRIS, the Institute for Robotics and Intelligent Systems (Canada's Network of Centers of Excellence). NASA Space Act Tech Brief Award (1991). The E. (Ben) \& Mary Hochhausen Award for Research in Adaptive Technology For Blind and Visually Impaired Persons (2002).

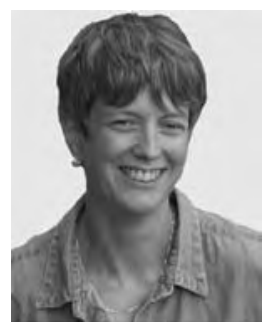

Karon E. MacLean received the BS degree in biology and mechanical engineering from Stanford University in 1986, and the MSc and $\mathrm{PhD}$ degrees from the Massachusetts Institute of Technology in mechanical engineering in 1988 and 1996, respectively. She worked for two years for the Center for Engineering Design at the University of Utah and, from 1996-2000, as a scientist at Interval Research in Palo Alto, California. She is currently an associate professor in the Department of Computer Science at the University of British Columbia, Vancouver, Canada, where her research focuses on haptic communication of information between people and intelligent systems. She is a member of the IEEE. 